\title{
A Case Report on Pyodermatitis-pyostomatitis vegetans (PD-PSV) in a patient with ulcerative colitis
}

\author{
Anushan $K^{1}$, Sivapirakasam $S^{2}$ \\ 1Colombo South Teaching Hospital, 2Teaching Hospital, Jaffna
}

\section{Introduction}

Pyodermatitis-pyostomatitis vegetans (PD-PSV)), is a very rare, chronic inflammatory dermatosis. It is characterized by large exudative, verrucous plaques with well-defined and elevated borders with typical mucocutaneous involvement. Cutaneous lesions can affect the genital and axillary areas, trunk as well as the scalp. Mucosal involvement is characterized by multiple pustules and vesicles on an erythematous base. Oral mucosa is mostly affected. PV(Pyoderma Vegetans) often affects middle-aged men. It is frequently associated with ulcerative colitis (UC), being considered a marker of inflammatory bowel disease (IBD). Predisposing factors include microbial infections, halogen ingestion, foreign materials and tattoos in patients with a compromised immune system.

In 1889 Hallopeu described clinical entity characterized by vegetating pustular lesions with benign outcome(1). McCarthy,in 1949, introduced the term pyostomatitis vegetants. To date some believe that it is a form of pemphigus, others, an idiosyncratic reaction (2).

Here, we present a case report of PV -PSD associated with UC and a short review of the literature on the therapeutic challenge of this rare disorder.

\section{Case Report}

A 73-year-old man presented to the dermatological clinic with a 2 month history of well-defined, vegetating plaques with elevated border in axilla, groin, flexural part of limbs and trunk with associated oral and nasal mucosal lesions were characterized by vesicles and pustules on an erythematous base (figure 1, $2 \& 3$ ). He had been on sulfasalazine for the last 2 years for UC.

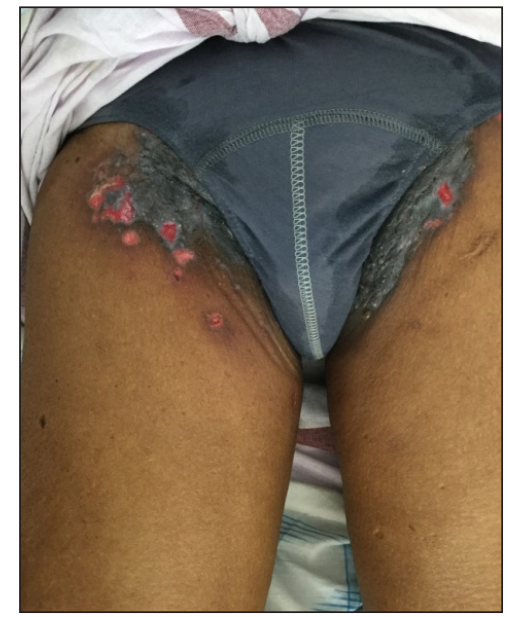

Fig 1

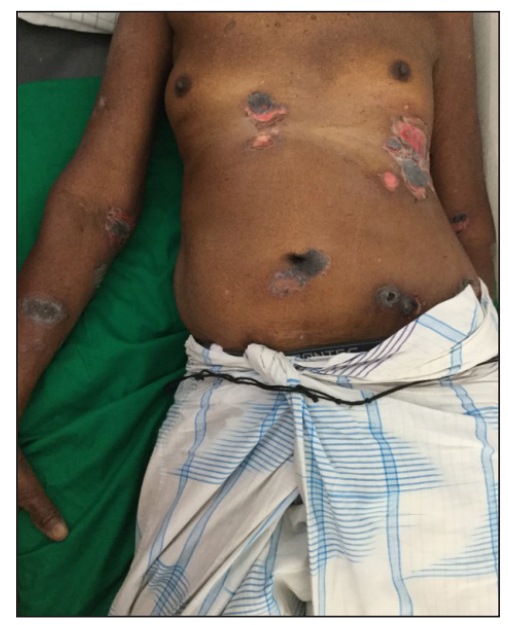

Fig 2

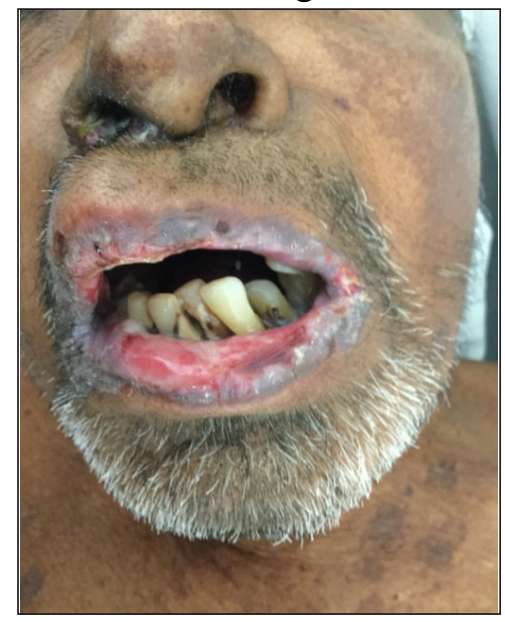

Fig 3 
Laboratory tests revealed a white blood cell count of $7.7 \times 10^{9} / 1$ neutrophils $55 \%$ with peripheral eosinophilia (10\%), hemoglobin $9.9 \mathrm{~g} / \mathrm{dl}, \mathrm{MCV}$ 93.5fl and Erythrocyte Sedimentation Rate of 62 $\mathrm{mm} / \mathrm{h}$, ALT 26IU/1, AST 27IU/1, total protein $63 \mathrm{~g} / \mathrm{dl}$ with Albumin $25 \mathrm{~g} / \mathrm{dl}$. Wound culture was positive for Staphylococcus aureus. Fungal culture was negative. Histopathological examination revealed papillamatous epidermal thickness, suprabasal separation of epidermis with crusts rich in eosinophil and occasional acantholysis cells. There were eosinophilic accesses in the epidermis (figure 4 \&5). Direct immunofluorescence test was negative. Based on these findings and clinical picture the diagnosis of PV-PSD was established.

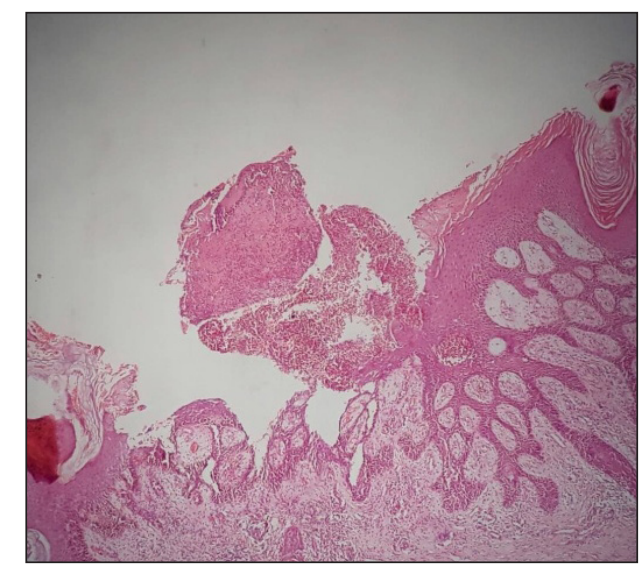

Figure 4

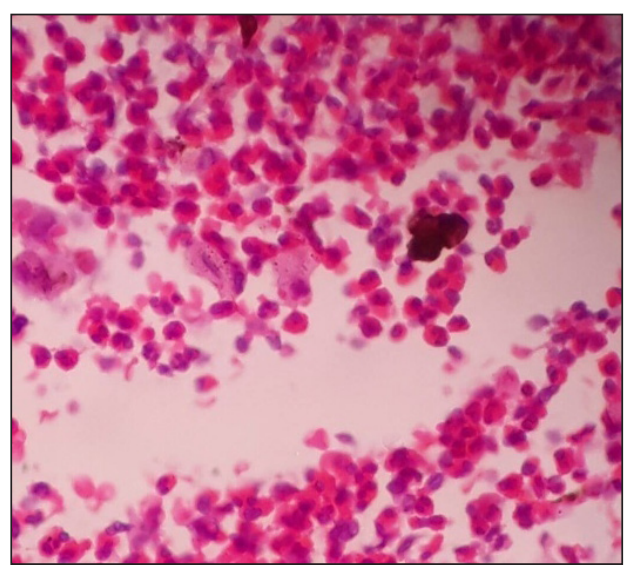

Figure 5

The patient was hospitalized with the working diagnosis of PV-PSD. Systemic administration of corticosteroids was started with prednisolone $60 \mathrm{mg}$ daily dose sulphasalazne $1 \mathrm{~g}$ bd with coamoxiclave $1.2 \mathrm{~g}$ bd for of bacterially colonized skin lesions. Topical wound care was given with antibiotic, antiseptic with steroid solutions. The skin lesions improved in months. Corticosteroids were gradually tapered and the patient was continued on sulfasalazine with a low maintenance dose of oral steroid.

\section{Discussion}

Pyodermatitis-pyostomatitis vegetans (PD-PSV) is a rare eosinophilic inflammatory dermatosis of unknown cause, characterized by vesicular and pustular lesions and vegetating plaques with mucocutaneous involvement (3). It generally occurs in individuals at the end of their third decade with a female male incidence ratio of $3: 1$ (4)

Cutaneous involvement is frequent, characterized by erythematous pustules papules, vesicles and lesions coated by crusts which coalesce into vegetating plaques. Lesions affect mainly axillae, scalp, vulva and groin. The oral mucosa is affected in most cases. Oral and nasal mucosal lesions characterized by vesicles and pustules on the erythematous base. Lips, gums, soft and hard palate, vestibule and tonsils are the most frequently affected areas. Vaginal, nasal and ocular mucosa may also be affected (5).

In our reported case the patient presented the characteristic cutaneous manifestations in typical areas. Clinical characteristics were similar to the ones described in the medical literature (6). Association of PD-PSV with IBD is well established. More commonly associated with ulcerative colitis (53\%) than Crohn's disease (11\%) of the patients (7).

Some authors suggest it as a spectrum of neutrophilic dermatoses and others propose that it represents a clinical manifestation of pyoderma gangrenosum. Some classify it as a hypersensitivity reaction (5).

Unlike PD-PSV, pemphigus vulgaris is potentially fatal and both the vegetans and vulgaris forms are well characterized as autoimmune bullous diseases revealing intercellular deposits of IgG and C3 by direct immunofluorescence. Histopathology of PDPSV is characterized by pseudoephiteliomatous hyperplasia with intraepithelial micro abscesses and eosinophilic spongiosis. Acantholysis and suprabasal clefts can be seen. In our case, histopathological findings were similar to those found in the medical literature.

Immunofluorescence differentiation between 
pemphigus vegetans and PD-PSV as this has both direct and indirect immunofluorescences negative. There is peripheral eosinophilia in $90 \%$ of the cases, having adiagnostic value.

The quick response to therapy with systemic corticosteroids is common in PD-PSV as observed in the reported case. Topical corticosteroids, sulfasalazine, oral prednisolone high dose gave the favorable prognosis. Prognosis for PD-PSV is excellent. Azathioprine and cyclosporine have already been used with success in previous case reports.

\section{References}

1. Hallopeau H. "Pyodermite vegetante". ihre Beziehungen zur dermatitis herpetiformis und dem pemphigus vegetans. Arch Dermatol Syphilol. 1898;43:289-306.
2. McCarthy P. Pyostomatitis vegetans: report of three cases. Arch Dermatol Syphilol. 1949;60:750-64

3. Elder DE, Elenitsas R, Johnson BL, Murphy GF, Xu X. Lever's Histopathology of the skin. 10th ed. Philadelphia: Lippincott Williams \& Wilkins, 2009. p. 546.

4. Degos R, Carteaud A. Pyodermite végétante de Hallopeau. Ann Dermatol Syph. 1953;80:254-62.

5. McCarthy P, Shklar G. A syndrome of pyostomatitis vegetans and ulcerative colitis. Arch Dermatolol. 1963;88:913-9.

6. Nigen S, Poulin $\mathrm{Y}$, Rochette L, Lévesque $\mathrm{MH}$, Gagné E. Pyodermatitis-pyostomatitis vegetans: two cases and a review of the literature. J Cutan Med Surg. 2003;7:250-5.

7. Leibovitch I, Ooi C, Huilgol SC, Reid C, James CL, Selva D. Pyodermatitis-pyostomatitis vegetans of the eyelids case report and review of the literature. Ophthalmology. 2005;112:1809-13. 